\title{
Síntomas emocionales y compras por pánico durante la pandemia de COVID-19: Un análisis de trayectoria
}

\section{Emotional Symptoms and Panic Buying during the COVID-19 Pandemic: A Path Analysis} \section{(D) Psicocente}
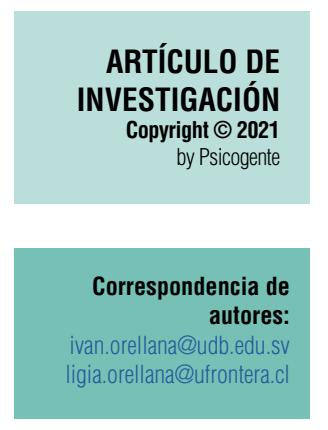

Recibido: 20-05-20 Aceptado: 20-01-21 Publicado: $30-01-21$

Carlos Iván Orellana iD
Universidad Don Bosco, Antiguo Cuscatlán, El Salvador
Ligia María Orellana iD
Universidad de La Frontera, Temuco, Chile

\section{Resumen}

Objetivo: Examinar las manifestaciones y los vínculos entre síntomas emocionales y las compras por pánico durante la aplicación de medidas de cuarentena domiciliar debido a la pandemia de COVID-19.

Método: Una muestra no probabilística de 339 residentes en El Salvador, mayores de 18 años, respondió un cuestionario en línea. El instrumento constaba de datos sociodemográficos, ítems sobre percepciones sobre la pandemia, una escala sobre compras por pánico y la escala abreviada de Depresión Ansiedad y Estrés (DASS-21). Se realizaron análisis bivariados y multivariados, incluyendo un análisis de trayectoria o path analysis.

Resultados: Casi $40 \%$ de la muestra incurrió en compras por pánico. Las compras por pánico se asociaron con depresión, ansiedad y estrés, con el interés en la pandemia y la percepción de alteración de rutinas. El análisis de trayectoria comprueba que, indirectamente, la depresión desalentó la compra por pánico y la ansiedad la incentivó. El estrés ejerció un efecto positivo indirecto a través del interés en el tema y la alteración de rutinas, pero un efecto directo sobre las compras por pánico.

Conclusión: La depresión, ansiedad y estrés se relacionan de manera diferencial con percepciones y comportamientos asociados al afrontamiento de la pandemia. El estrés constituye una respuesta adaptativa a la demanda de la situación mientras que las compras por pánico son una forma de paliar las emociones negativas suscitadas por la crisis. El interés en estos patrones de compra debe contemplar el contexto consumista en el que ocurren, así como las formas de afrontamiento en función del poder adquisitivo.

Palabras clave: compras por pánico; depresión; estrés; ansiedad; COVID-19; análisis de trayectoria

\section{Abstract}

Objective: To examine the manifestations and links between emotional symptoms and panic buying during the implementation of domiciliary quarantine measures due to the COVID-19 pandemic.

Method: A non-probabilistic sample of 339 residents in El Salvador, over 18 years old, answered an online questionnaire. The instrument comprised sociodemographic questions, items on perceptions of the pandemic, a panic buying scale, and the short version of the Depression, Anxiety and Stress Scale (DASS-21). Bivariate and multivariate analyzes were performed, including a path analysis.

Results: Almost $40 \%$ of the sample engaged in panic buying. Panic buying was associated with depression, anxiety, and stress, with being interested in the pandemic and the perception of disrupted routines. The path analysis showed that, indirectly, depression discouraged panic buying and anxiety encouraged it. Stress exerted an indirect positive effect mediated by the interest in the pandemic and the disruption of routines, but a direct effect on panic purchases.

Conclusion: Depression, anxiety, and stress are differentially related to perceptions and behaviors associated with coping with the pandemic. Stress is an adaptive response to the demands of the situation while panic buying is a way to alleviate the negative emotions caused by the crisis. The interest in these shopping patterns must contemplate the consumerist context in which they occur as well as coping strategies linked to acquisitive power.

Keywords: panic buying; depression; anxiety; stress; COVID-19; path analysis 


\section{INTRODUCCIÓN}

Uno de los fenómenos que más ha captado la atención mediática en el marco del avance de la pandemia de COVID-19 a nivel global (Organización Mundial de la Salud, OMS, 2020), ha sido el de las denominadas compras por pánico (panic buying). El pánico alude a un "miedo colectivo intenso" y connota acciones primitivas, desordenadas y hasta violentas que se producen en el contexto de una catástrofe (Martín Beristain, 1999, p.67). Para el caso, la pandemia y las incertidumbres que suscita. Las compras por pánico constituyen comportamientos de consumo que se concretan en aglomeraciones relativamente espontáneas en supermercados, que persiguen obtener artículos que se consideran "de primera necesidad" ante la situación en desarrollo. El aspecto perceptual o interpretativo implicado es relevante, porque la atribución de primera necesidad que recibe un artículo no siempre encuentra una justificación lógica, como ha demostrado la compra masiva de papel higiénico que tanta notoriedad ha tenido en diferentes partes del mundo.

El carácter global de este patrón de consumo en diferentes partes del planeta debido al avance de la pandemia justifica considerar las compras por pánico como un fenómeno de psicología de masas (Yap \& Chen, 2020). Aún más, como sostiene Novemsky (2020, párr. 1), las compras por pánico encontrarían mucho de su explicación en "una tormenta perfecta de fuerzas psicológicas". Precisamente, lejos de la existencia de un único factor explicativo a la base de las compras por pánico, su ocurrencia parece responder a la activación simultánea de presiones psicológicas y fuerzas situacionales propiciadas por la circunstancia total y amenazante que configura la pandemia.

La pandemia, como coinciden varios autores (Norberg \& Rucker, 2020; Novemsky, 2020; Sim, Chua, Vieta \& Fernandez, 2020; Taylor, 2019; Urzúa, Vera-Villarroel, Caqueo-Urízar \& Polanco-Carrasco, 2020; Yap \& Chen, 2020), constituye una situación marcada por la incertidumbre, comportamientos, reacciones y síntomas psicológicos varios, la percepción de escasez y la sensación de pérdida de control. Es decir, la imposibilidad súbita e impuesta para provocar cambios a voluntad en el medio o para mantener lo que hasta hace poco eran rutinas propias e ininterrumpidas (Yap \& Chen, 2020). Asimismo, la naturaleza de cada pandemia impulsa a la búsqueda de ciertos productos y no de otros (Martín Beristain, 1999; Norberg \& Rucker, 2020). Dado el conocimiento general sobre la COVID-19 y las formas de prevenir su contagio (e.g., lavado de manos, portar mascarillas), aquellos productos presumiblemente más útiles (e.g., desinfectantes, lejía) serán los más 
buscados. Esta demanda se basa en la atribución de valor o efectividad que las personas asignan a estos productos por -supuestamente- resolver el problema u ofrecer protección a los seres queridos, cuestión que además conlleva experimentar afectos positivos a pesar de las circunstancias adversas (Folkman \& Moskowitz, 2000).

No obstante, como fue mencionado, las compras por pánico, u otros comportamientos como la acumulación, se tratan más de comportamientos de masas que mezclan aspectos racionales con aspectos irracionales y emocionales (Sheu \& Kuo, 2020). Con el trasfondo de la incertidumbre y el miedo por la pandemia, la asistencia masiva a los supermercados, la proliferación de estas escenas en los medios de comunicación, contemplar el agotamiento de productos y ver imágenes de estantes vacíos, dispara presiones de grupo, modelaje y comportamientos conformistas de rebaño (Sim et al., 2020; Yap \& Chen, 2020). Así, cada vez más gente llega a los lugares de compra mientras en el lugar cada vez más personas buscan hacerse de productos que otros están adquiriendo. La situación, entonces, se agrava porque induce una profecía que se cumple por sí misma: el miedo a la escasez de productos justamente produce su agotamiento vía la compra masiva y simultánea de los mismos (Yap \& Chen, 2020). Se trata del clásico proceso de "la tragedia de los comunes" en el que la competición por recursos finitos termina por eliminarlos, pero escenificado en el pasillo de un supermercado.

Varios heurísticos y sesgos cognitivos -es decir, atajos y distorsiones sistemáticas de pensamiento y evaluación- alimentan situacionalmente las compras por pánico. Por ejemplo, la percepción de escasez conlleva mayor atribución de valor a los productos, incrementando la necesidad creada de obtenerlos. Mientras se contempla que otros adquieren cosas que uno no tiene, emerge la aversión a la pérdida, la desagradable sensanción que acompaña al quedarse con las manos vacías, creer que no se ha logrado lo suficiente o lo que otros sí han obtenido, y que hace incurrir en grandes esfuerzos (e.g., hacer largas colas) y costos (e.g., pagar de más, exponerse al contagio) para contrarrestar dicha sensación (Brafman \& Brafman, 2008; Novemsky, 2020).

Asimismo, la pandemia constituye una circunstancia que destaca la mortalidad, incluyendo la propia. Así, se propician motivaciones que persiguen reducir la ansiedad aparejada: unas defensivas, como aferrarse a las propias visiones de mundo, y otras motivaciones centradas en causar una buena impresión que devuelvan la sensación de valía personal. Las motivaciones defensivas incluyen el propio interés material y las que buscan causar buena impresión conllevan someterse a la presión de grupo en procura de recobrar 
autoestima. Ambas motivaciones por tanto, fruto de la combinación del ambiente de amenaza, mortalidad y ansiedad, resultan compatibles con comportamientos colectivos de consumo (Maheswaran \& Agrawal, 2004), como las compras por pánico. Esta cadena de circunstancias, procesos grupales y de pensamiento y la letalidad ansiógena que acompañan a la pandemia, junto con el tamaño y la visibilidad de los paquetes de papel higiénico, explicarían en buena medida el curioso pero irracional atractivo que este producto ha recibido por parte de los consumidores en el marco de la pandemia por COVID-19.

No obstante, si bien estados emocionales y el consumo por pánico se encuentran vinculados, su asociación es variable. En el contexto de incertidumbre y amenaza generalizada propio de una pandemia, la compra por pánico parece funcionar como una estrategia de afrontamiento adaptativo frente al estrés enfocado en el problema (Folkman \& Moskowitz, 2000; Sim et al., 2020; Yap \& Chen, 2020). La activación por estrés (stress arousal) constituiría una condición preliminar o de partida que busca adaptarse a la demanda del medio o, de lo contrario, podría dar pie a otras consecuencias perniciosas, incluyendo la ansiedad y la depresión (Dar, lqbal \& Mushtaq, 2017; Everly \& Lating, 2013; Ruiz, García-Martín, Suárez-Falcón \& Odriozola-González, 2017). La ansiedad respondería más a compras situacionales y compulsivas (Gallagher, Watt, Weaver, \& Murphy, 2017), mientras la depresión parece fomentar conductas de acumulación, de percepción de ralentización temporal o aferramiento al pasado e ideación y comportamientos autoderrotistas (Grisham et al., 2018; Thomson, 2016; Zimbardo \& Boyd, 2009). Las relaciones unívocas entre estados emocionales y comportamientos colectivos de consumo deben ser tomadas con reserva, porque las investigaciones difieren respecto a la forma en que operacionalizan los unos (i.e., emplean diferentes escalas de medición para constructos que se denominan igual) y el otro (i.e., en condiciones estables o de alarma social, en jóvenes, distintas culturas).

Por otra parte, al estudiar los disparadores emocionales de las compras por pánico hay que considerar el contexto de emergencia en desarrollo, pero también el trasfondo neoliberal consumista en que estas tienen lugar y que propende a la búsqueda de estatus y bienestar a través de la adquisición perenne de cosas innecesarias (Norberg \& Rucker, 2020). Igualmente, en cada tiempo y lugar la depresión, la ansiedad y el estrés exhiben una prevalencia peculiar previa al brote de coronavirus. Justamente, en caso de El Salvador, se observaron manifestaciones de compras por pánico con las 
primeras noticias del avance de la pandemia y existen indicios de la prevalencia de síntomas emocionales en su población. En este país centroamericano, cuando aún no se reportaban casos conocidos en su territorio, se registraron afluencias multitudinarias a supermercados, compras a granel de artículos de limpieza, y la implementación de medidas restrictivas de control por parte de los establecimientos, tales como la limitación del número de artículos adquiribles o avisos de abastecimiento suficiente (La Prensa Gráfica, 2020; Molina, 2020). Al mismo tiempo, replicando tendencias mundiales, en dicho país la depresión y la ansiedad constituyen condiciones comórbidas, altamente prevalentes, "comunes" y que parecen ser más agudas en el caso de las mujeres (Gutiérrez Quintanilla \& Portillo García, 2013; Ministerio de Salud de El Salvador, MINSAL, 2017; OPS \& OMS, 2017).

La variabilidad del vínculo entre estados emocionales y conductas de consumo, la peculiaridad de las compras por pánico en el contexto de una pandemia y el escaso conocimiento de estos aspectos en un contexto como el salvadoreño, justifican llevar a cabo este estudio. Por tanto, el objetivo general de esta investigación fue explorar la manifestación y la asociación existente entre los estados emocionales de depresión, ansiedad y estrés y las compras por pánico en población salvadoreña en el marco de la pandemia por COVID-19.

\section{MÉTODO}

\subsection{Participantes}

La muestra se obtuvo a través de un muestreo intencional propositivo (Clark-Carter, 2002). Los participantes fueron 339 personas residentes en El Salvador, de 18 años o más, quienes respondieron a un cuestionario en línea que se mantuvo abierto mientras permanecía vigente el estado de excepción y la cuarentena domiciliar debido a la pandemia de COVID-19 en el país. La muestra final estuvo compuesta por participantes de ambos géneros (62 \% mujeres y $38 \%$ hombres). Un 52,8 \% de los participantes reportó trabajar; $20,4 \%$ dedicarse tanto a trabajar como a estudiar; $18 \%$ de la muestra reportó que estudiaba y el restante $8,8 \%$ que se dedicaba a otras cosas (e.g., oficios del hogar, buscar empleo). El $26 \%$ de participantes afirmó que su ingreso económico es suficiente en su hogar y le permite ahorrar; 51,6 \% afirmó que el ingreso alcanza y no enfrenta grandes dificultades; el 22,4 \% restante reportó que el ingreso mensual en el hogar no le alcanza y cada mes experimentaría cierto grado de dificultad económica. 


\subsection{Instrumento}

\subsubsection{Datos sociodemográficos}

Esta primera sección del cuestionario registró los datos sociodemográficos descritos en el apartado anterior. La pregunta sobre ingreso en el hogar proviene de los sondeos de cultura política realizados por el Proyecto de Opinión Pública de América Latina (LAPOP, ver Córdova Macías, Rodríguez y Zechmeister, 2017).

\subsubsection{Escala sobre acciones y percepciones en el marco de la pandemia} (elaboración propia)

Otro bloque de preguntas estuvo compuesto por ítems referidos a ciertas percepciones vinculadas con la pandemia con respuesta tipo Likert de cuatro puntos, $1=$ Nada, 4 = Mucho: cuatro ítems conformaron una escala de interés en la pandemia: la enfermedad misma, seguimiento de noticias, el acatamiento de medidas higiénicas y el temor a infectarse $(\alpha=0,62)$. Otros cuatro ítems dieron forma a una escala de alteración de rutinas en el marco de la pandemia y la cuarentena domiciliar: incremento de uso de internet o teléfono móvil, alteración de rutina de trabajo u oficios, en el hogar y la percepción de deterioro de la calidad de las relaciones en el hogar $(\alpha=0,63)$. Los ítems creados y su agrupación para formar las escalas mencionadas se muestran en la Tabla 1.

Tabla 1.

Formulación de ítems que conforman las escalas creadas de acciones y percepciones

\begin{tabular}{c} 
INTERÉS EN EL TEMA DE LA PANDEMIA: \\
\hline ¿Ha procurado enterarse sobre qué es el coronavirus? \\
¿Ha dado seguimiento a las noticias sobre el COVID-19? \\
¿Ha procurado seguir las recomendaciones sanitarias recomendadas? \\
Dada su circunstancia, ¿le preocupa llegar a infectarse? \\
\hline ALTERACIÓN DE RUTINAS: \\
\hline ¿El uso de su teléfono móvil o internet ha incrementado estos días? \\
¿Su rutina de estudio o trabajo (o jubilación, oficios, etc.) se ha visto alterada? \\
¿Su rutina habitual en el hogar se ha visto alterada? \\
¿La calidad de las relaciones en el hogar se ha visto deteriorada?
\end{tabular}

\subsubsection{Escala de compras por pánico (elaboración propia)}

La escala de compras por pánico creada para este estudio se compone de cinco ítems con estructura de respuesta dicotómica ( $1=\mathrm{Sí}, 0=\mathrm{No})$ que aluden a acciones propias de este tipo de consumo masivo. Se pregunta por la compra en días desacostumbrados para hacerlo, por compras a pesar de conocer de las aglomeraciones, aun sabiendo que no habrá desabastecimiento y la adquisición inusual de artículos de limpieza y de alimentos. Se 
llevó a cabo un análisis factorial exploratorio (AFE) luego de corroborar que los datos eran adecuados para un análisis de estas características (KMO = 0,73 y prueba de esfericidad de Bartlett con valor de $p=0,000$ ). Recurriendo a un método de componentes principales, el análisis mostró la existencia de un factor único (compras por pánico), que daba cuenta del 45,22 \% de la varianza de los resultados. La consistencia interna de la escala se situó apenas por debajo del límite inferior convencional satisfactorio de 0,70 ( $\alpha=$ $0,69)$. No obstante, cabe considerar el alfa alcanzado como suficiente, dada la brevedad de la escala, la estructura binaria de sus opciones de respuesta, los fines exploratorios perseguidos y que el simple aumento de ítems incrementaría el coeficiente obtenido (ver Taber, 2018). El enunciado de los ítems de la escala se presenta en el apartado de resultados.

\subsubsection{Escala abreviada de Depresión, Ansiedad y Estrés (DASS-21) (Antúnez \& Vinet, 2012; Clinik Lab, s.f.)}

EI DASS está compuesto por 21 ítems, siete ítems por cada factor de primer orden (depresión, ansiedad y estrés), y que a la vez puede analizarse como una medida de síntomas emocionales o afectividad negativa como factor de segundo orden (Ruiz et al., 2017). Las opciones de respuesta en esta escala son cuatro y van de $0=$ "No me ha ocurrido" a 3 = "Me ha ocurrido mucho, o la mayor parte del tiempo". Lo que indica que el mayor puntaje conlleva mayor frecuencia de experimentación de síntomas emocionales. La validez y la confiabilidad de la escala y sus subdimensiones ha sido comprobada en diversas muestras de habla hispana (Antúnez \& Vinet, 2012; Apóstolo, Figueiredo, Mendes \& Rodrigues, 2011; García-Rivera, Maldonado-Radillo \& Ramírez Barón, 2014; Román, Vinet \& Alarcón Muñoz, 2014; Ruiz et al., 2017). El análisis del DASS-21 en este estudio se basó en la propuesta del Laboratorio de Psicología Clínica (Clinik Lab, s.f.), de la Fundación Universitaria Konrad Lorenz de Colombia referida a sus ítems, sus opciones de respuesta y los criterios de interpretación. La consistencia interna de la escala y sus dimensiones fue satisfactoria, con un Alfa de Cronbach de 0,95 para la escala general y un alfa de $0,90,0,88$ y 0,88 para depresión, ansiedad y estrés, respectivamente.

\subsection{Procedimiento}

El cuestionario fue activado el 18 de marzo de 2020 y se mantuvo activo hasta el 5 de abril, mientras permanecían vigentes medidas de restricción de movimiento debido a la pandemia en El Salvador. El cuestionario fue distribuido en línea y el reclutamiento de participantes se realizó a través de un proceso de bola de nieve. La página inicial del cuestionario identificó a esta investigación como un "Estudio sobre salud mental durante la cuarentena 
nacional por COVID-19" y exponía los criterios de inclusión (residir en El Salvador y ser mayor de 18 años). Se describió también la estructura del cuestionario, señalando que el formato de respuesta era cerrado y su llenado tomaba menos de 10 minutos. Se solicitó el consentimiento de los participantes a responder la encuesta, garantizando el anonimato, la confidencialidad, el uso estrictamente académico de la información y la libertad de abandonar el llenado en cualquier momento. De 343 cuestionarios recibidos, se descartaron cuatro, pues tal cantidad de participantes no cumplía con el requisito de la edad.

\subsection{Análisis de datos}

El cuestionario fue construido en la plataforma Google Forms. La base de datos obtenida se transformó y analizó con los programas SPSS v.25. y AMOS v.23. El SPSS sirvió para llevar a cabo exploraciones, recodificaciones, construcción de escalas y análisis estadísticos descriptivos e inferenciales bivariados y multivariados. AMOS fue utilizado para elaborar el análisis de trayectoria (Path Analysis), un modelo que pretende vincular dinámicamente los síntomas emocionales y las compras por pánico.

\section{RESULTADOS}

En la Tabla 2 se muestra la proporción de respuesta afirmativas y negativas que permiten aproximarse a la magnitud de las compras por pánico realizadas por los participantes en el estudio.

Tabla 2.

Manifestación de compras por pánico en porcentajes

\begin{tabular}{|c|c|c|}
\hline ÍTEMS & sí & NO \\
\hline ¿Realizó compras en días en que no las haría en circunstancias normales? & $57,2 \%$ & $42,8 \%$ \\
\hline ¿Realizó compras en días en que no las haría en circunstancias normales, a pesar de la aglomeración de personas? & $31,9 \%$ & $68,1 \%$ \\
\hline $\begin{array}{c}\text { ¿Realizó compras en días en que no las haría en circunstancias normales, a pesar de que fue anunciado que no habría } \\
\text { desabastecimiento? }\end{array}$ & $34,5 \%$ & $65,5 \%$ \\
\hline ¿Compró cantidades o tipos de artículos de limpieza o higiene que no compraría en circunstancias normales? & $30,7 \%$ & $69,3 \%$ \\
\hline ¿Compró cantidades o tipos de alimentos que no compraría en circunstancias normales? & $40,4 \%$ & $59,6 \%$ \\
\hline Promedio & $38,9 \%$ & $61,1 \%$ \\
\hline
\end{tabular}

Los resultados expuestos en la Tabla 2 corroboran que casi cuatro de cada 10 participantes incurrió en comportamientos propios de compras por pánico. Asimismo, las acciones de compras por pánico que más destacan entre las consignadas son la asistencia al lugar de compras en días desacostumbrados $(57,2 \%)$, seguida de la adquisición inusual de cantidades o tipos alimentos $(40,4 \%)$. 
Por otro lado, los niveles y las asociaciones relevantes entre las variables de estudio se presentan en la Tabla 3.

Tabla 3.

Estadísticos descriptivos y correlaciones relevantes entre la escala de compras por pánico, síntomas emocionales y variables de control.

\begin{tabular}{ccccccccccc}
\hline & & $\mathbf{( 1 )}$ & $\mathbf{( 2 )}$ & $\mathbf{( 3 )}$ & $\mathbf{( 4 )}$ & $\mathbf{( 5 )}$ & $\mathbf{( 6 )}$ & $\mathbf{( 7 )}$ & $\mathbf{( 8 )}$ & (9) \\
\hline$(1)$ & Compras por pánico & - & & & & & & & & \\
$(2)$ & Depresión & $0,17^{* *}$ & - & & & & & & \\
$(3)$ & Ansiedad & $0,22^{* *}$ & $0,74^{* *}$ & - & & & & & \\
$(4)$ & Estrés & $0,32^{* *}$ & $0,78^{* *}$ & $0,79^{* *}$ & - & & & & \\
$(5)$ & Interés en el tema & $0,23^{* *}$ & 0,11 & $0,26^{* *}$ & $0,24^{* *}$ & - & & & \\
$(6)$ & Alteración de rutinas & $0,32^{* *}$ & $0,27^{* *}$ & $0,28^{* *}$ & $0,39^{* *}$ & $0,14^{*}$ & - & & \\
$(7)$ & Ingreso en el hogar & $-0,09$ & $0,12^{*}$ & 0,03 & $-0,03$ & 0,03 & $-0,02$ & - & \\
$(8)$ & Ocupación & $-0,04$ & $-0,23^{* *}$ & $-0,11$ & $-0,12^{*}$ & 0,04 & $-0,16^{* *}$ & $-0,17^{* *}$ & - & \\
$(9)$ & Sexo & 0,09 & 0,06 & $0,14^{*}$ & $0,15^{*}$ & 0,04 & 0,09 & 0,06 & 0,05 & - \\
& $\mathrm{M}$ & 2,0 & 4,3 & 3,6 & 6,3 & 14,0 & 11,6 & 2,0 & - & - \\
- & $\mathrm{DE}$ & 1,6 & 5,0 & 4,2 & 4,8 & 1,9 & 2,7 & 0,7 & - & - \\
\hline
\end{tabular}

${ }^{*} p<0,05, * * p<0,01$

Abreviaturas: $\mathrm{M}=$ Media aritmética, $\mathrm{DE}$ = Desviación estándar. Codificación de variables: compras por pánico: escala de 0-5 puntos (puntajes tendientes a 5 refieren más comportamientos propios de compras por pánico); depresión, ansiedad y estrés: cada dimensión corresponde a una escala de 0-21 puntos $(0=$ No me ha ocurrido, $21=$ Me ha ocurrido mucho, o la mayor parte del tiempo); interés en el tema: escala de 1-16 puntos (puntajes mayores indican mayor interés en el tema); alteración de rutinas: escala de 1-16 puntos (puntajes mayores indican mayor alteración diaria de rutinas); ingreso en el hogar: 1 = alcanza y puede ahorrar, 2 = alcanza justo y sin grandes dificultades, $3=$ no alcanza y tiene dificultades, 4 = no alcanza y tiene grandes dificultades; ocupación es una variable ficticia o dummy: 1 = trabaja, $0=$ otros (estudia, jubilado, oficios del hogar, etc.); sexo: $1=$ mujer, $0=$ hombre.

La Tabla 3 corrobora que, en promedio, los participantes llevaron a cabo dos acciones propias de compras por pánico (de cinco consideradas). Asimismo, las compras por pánico correlacionan positivamente con la depresión, la ansiedad, el estrés, el interés en el tema y la alteración de rutinas cotidianas $(0,25$ en promedio, $p<0,001)$, pero no correlacionan con ninguna de las variables de control consideradas. Los síntomas emocionales presentan promedios que los califican como leves y se encuentran alta y fuertemente correlacionados de manera directa. La depresión correlaciona positivamente con la alteración de rutinas y con un ingreso económico deficitario, y correlaciona negativamente, aunque fuertemente, con no trabajar (estudiar, estar jubilado, dedicarse a oficios en el hogar, etc.). La ansiedad y el estrés correlaciona positivamente con ser mujer, y padecerían más estrés también quienes al momento de realizar el estudio reportaron no trabajar. Los promedios de interés en el tema y de alteración de rutinas son altos $(14,0$ y 11,6 , respectivamente), y ambas variables se encuentran directamente relacionadas, aunque débilmente. La percepción de alteración de rutinas sería más acusada en quienes no trabajan y quienes reportaron un ingreso mensual que daba dificultades a fin de mes. 
Para estimar la relación dinámica entre las compras por pánico y los síntomas emocionales, se elaboró un análisis de trayectoria o path análisis con las variables presentadas en la Tabla 3, en el que las compras por pánico constituyen la variable dependiente del modelo. La calidad del modelo fue establecida considerando varios índices de bondad de ajuste (i.e, índices de ajuste absoluto, índices de ajuste comparativo) bajo la premisa que es más probable contar con un modelo con buen ajuste entre más índices muestren un ajuste apropiado (Schreiber, Nora, Stage, Barlow \& King, 2006). Los siguientes valores son considerados como indicadores de un modelo con buen ajuste: $\chi 2 \mathrm{p}=$ n.s., $\chi 2 / \mathrm{df}<2,0, \mathrm{NFI}>0,90$, RFI cercano a 0,95, IFI-TLI-CFI $>0,95$, RMR cercano a 0, y RMSEA < 0,06 (Hooper, Coughlan \& Mullen, 2008; Schreiber et al., 2006). Con base en estos nueve criterios, se puede afirmar que el modelo obtenido cuenta con ajuste apropiado: $\chi 2 \mathrm{p}=0,22, \chi 2 / \mathrm{df}=$ $1,41, \mathrm{NFI}=0,99, \mathrm{RFI}=0,97, \mathrm{IFI}=0,99, \mathrm{TLI}=0,99, \mathrm{CFI}=0,99, \mathrm{RMR}=0,20$; $\mathrm{RMSEA}=0,04$. El diagrama del modelo del análisis de trayectoria se muestra en la Figura 1.

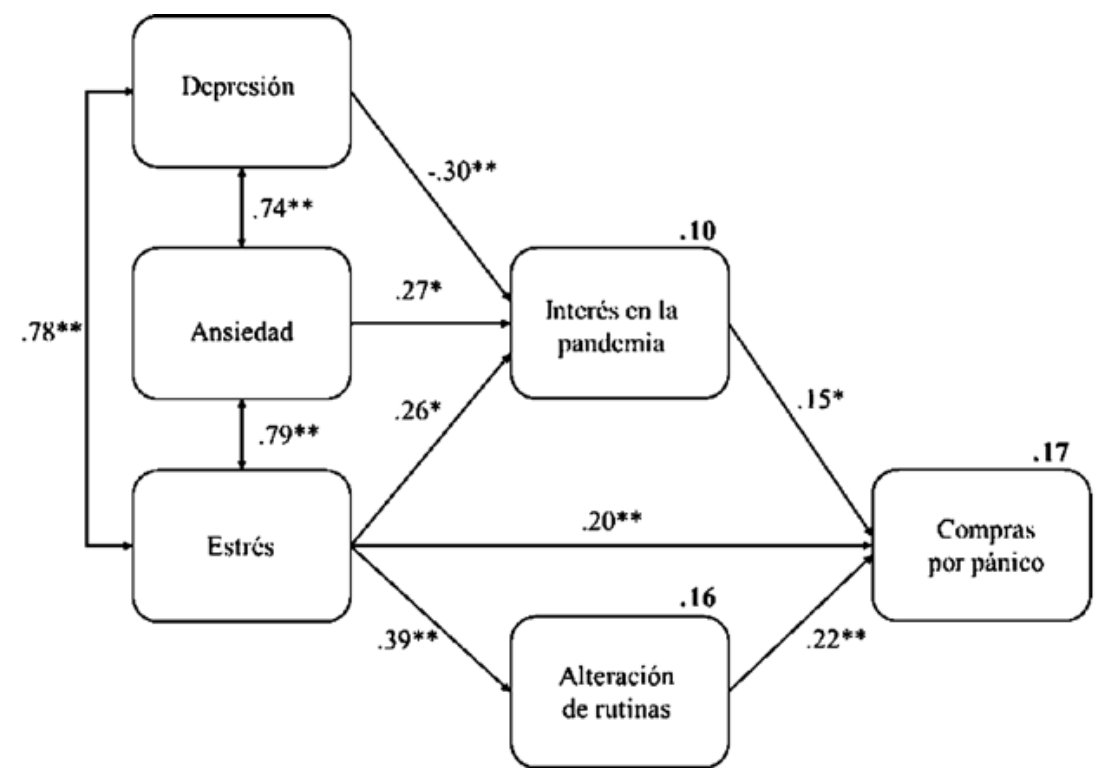

Figura 1.

Diagrama de trayectoria de síntomas emocionales y compras por pánico

Nota. Los números constituyen coeficientes estandarizados, ${ }^{*} p<0,01, * * p<0,001, \mathrm{~N}=339$. El diagrama solo presenta trayectorias significativas: las flechas de doble punta ilustran correlaciones entre las variables exógenas, las de una punta que se dirigen hacia las variables endógenas constituyen regresiones y los valores que las acompañan valores de coeficientes $\beta$. Los números en negritas refieren varianza explicada $\left(R^{2}\right)$. Las variables de control (sociodemográficas) y las variables endógenas de error no se muestran para mostrar un diagrama más limpio.

El modelo parte de asumir la relación comórbida que existe entre la depresión, la ansiedad y el estrés, un caldo de cultivo de afectos negativos que, propiciados por la circunstancia, instigan mayor interés en la pandemia 
(i.e., el coronavirus y sus formas de contagio, noticias) y la percepción de alteración de rutinas (e.g., trabajo, incremento de uso de internet) propiciada por las medidas implementadas para prevenir su avance. Estas percepciones y el estrés ejercerían un efecto directo sobre la inclinación a llevar a cabo compras por pánico, así como de forma indirecta lo harían la depresión, la ansiedad y el estrés a través del interés en el tema, y este último también por medio de la alteración de rutinas. Todos los vínculos (correlaciones y regresiones) que presenta la Figura 1 son estadísticamente significativos, mientras las variables de control asociadas a las compras por pánico no arrojaron resultados significativos.

Como se muestra en la Tabla 3, las correlaciones entre la depresión, la ansiedad y el estrés son altas y fuertes $(r=0,77$ en promedio, $p<0,001)$. En relación a las trayectorias (efectos directos) que se desprenden de la depresión y la ansiedad hacia el interés en el tema, aparece una relación inversa. La depresión se traduciría en desinterés en el tema y la ansiedad conllevaría más interés en el tema (respectivamente, $\beta=-0,30$ y $\beta=0,27$, $p<0,001)$ al igual que el estrés $(\beta=0,26, p<0,001)$, influjos que, en conjunto, darían cuenta del $10 \%$ de la varianza del interés en la pandemia. Luego, el estrés también ejercería un efecto directo positivo -el más fuerte por ciertosobre la percepción de alteración de rutinas $(\beta=0,39, p<0,001, R 2=0,16)$. y un efecto similar sobre las compras por pánico $(\beta=0,20, p<0,001)$. El interés en la pandemia y la alteración de rutinas igualmente se relacionan de forma directa y positiva con las compras por pánico (respectivamente, $\beta=0,15$ y $\beta=0,22, p<0,001)$.

El modelo también presenta efectos indirectos, débiles pero significativos. Cada síntoma emocional ejerce un efecto indirecto sobre las compras por pánico por medio del interés en la pandemia. El efecto indirecto de la depresión sobre las compras por pánico es negativo $(\beta=-0,05, p=0,01)$ y el de la ansiedad es positivo $(\beta=0,04, p=0,04)$. El estrés, además del efecto directo antes apuntado, ejerce un efecto indirecto combinado sobre las compras por pánico mediado tanto por el interés en el tema de la pandemia como por la alteración de rutinas que alcanza un valor de $\beta=0,13(p=0,03)$. Finalmente, cabe señalar que el modelo construido explica el $17 \%$ de la varianza de las compras por pánico.

\section{DISCUSIÓN}

El estudio se planteó como objetivo general explorar la manifestación y la relación de ciertas variables con las compras por pánico durante el desarrollo 
de la pandemia de COVID-19. Los resultados obtenidos ofrecen evidencia que las compras por pánico encuentran asociaciones particulares con percepciones que se producen durante la pandemia y con síntomas emocionales como la depresión, la ansiedad y el estrés.

Las compras por pánico responden a la convergencia de una plétora de factores y procesos de distinto nivel. Racionales e irracionales, motivacionales, cognitivos y emocionales, que a su vez se ven alimentados por presiones situacionales particulares y comportamientos de masa que tienen lugar en condiciones macrosociales de incertidumbre propios de la situación de pandemia (Brafman \& Brafman, 2008; Sheu \& Kuo, 2020; Yap \& Chen, 2020). De ahí que la enorme dificultad a la hora de estudiar este fenómeno, además de su ocurrencia esporádica y relativamente espontánea, sea lograr capturar las condiciones contextuales, situacionales y la "tormenta perfecta" de fuerzas psicológicas que entran en juego (Novemsky, 2020).

Se encontró que un $40 \%$ de la muestra habría realizado, en promedio, al menos dos comportamientos propios de compras por pánico: compras en días no acostumbrados, a pesar de la aglomeración, aunque no existía desabastecimiento o habría adquirido tipos o cantidades inusuales de artículos de limpieza o de alimentos. El carácter inusitado del patrón de consumo que además tiene lugar en condiciones de incertidumbre, incomodidad y suministro anunciado y suficiente de productos, sugiere que se trata de un comportamiento donde la interpretación peculiar de la situación (e.g., escasez) o la atribución utilitarista o de "primera necesidad" de los artículos, juegan un papel importante (Norberg \& Rucker, 2020; Sim et al., 2020).

Las correlaciones encontradas muestran que la depresión, la ansiedad y el estrés se asocian positiva y fuertemente entre sí, y cada una de estas dimensiones emocionales con las compras por pánico. Las compras también encuentran relación con el interés en el tema y la alteración de rutinas. Estas asociaciones ofrecen indicios del carácter comórbido de los síntomas emocionales en sí mismo y durante la pandemia, pero también que las compras por pánico responden al influjo simultáneo y complejo de diferentes disposiciones psicológicas cognitivas y emocionales (Dar et al., 2017; Novemsky, 2020; OPS \& OMS, 2017).

No obstante, la naturaleza diferencial de los síntomas emocionales se pone de manifiesto al constatar que la depresión no se relaciona con el interés en el tema, que la ansiedad y el estrés no se vinculan estadísticamente con contar con menos ingresos en el hogar o que no trabajar tampoco se asocia 
con la ansiedad, pero sí con la depresión y el estrés. Por otro lado, como la evidencia sostiene, las mujeres presentaron niveles significativamente más altos de ansiedad y estrés que los hombres, aunque el género no estableció diferencias respecto a la depresión (Gutiérrez Quintanilla \& Portillo García, 2013; MINSAL, 2017). No obstante, es en los análisis multivariados del análisis de trayectoria que aparecen pistas más claras del influjo diferencial de los síntomas emocionales y de las percepciones sobre las compras por pánico.

El modelo construido cuenta con un buen ajuste y ofrece evidencia de la relación entre síntomas emocionales, percepciones a propósito de la pandemia y las compras por pánico. Además de las correlaciones entre los síntomas emocionales que ya han sido mencionadas, por un lado, se encontró un efecto directo de la depresión, la ansiedad y el estrés sobre el interés en la pandemia. Lo interesante es que mientras la ansiedad y la depresión ejercen un efecto positivo, el efecto de la depresión resulta negativo, al igual que su efecto indirecto sobre las compras por pánico. En otras palabras, coincidiendo con la teoría (Everly \& Lating, 2013; Grisham et al., 2018; Zimbardo \& Boyd, 2009), según los resultados obtenidos, el estrés y la ansiedad alientan el comportamiento, pero la depresión lo desalienta (i.e., informarse sobre el COVID-19, seguir noticias, acudir a realizar compras). Quiere decir que aquellas personas que experimentan síntomas depresivos serían las más renuentes a seguir las novedades en torno a la pandemia, tenderían a retraerse o dudar de la efectividad de sus acciones (Thomson, 2016) o quizás, tenderían a acumular productos (Grisham et al., 2018), un comportamiento posterior a la compra, pero de carácter más bien pasivo.

La ansiedad directa e indirectamente-mediada por el interés en la pandemiaejerce un efecto positivo sobre las compras por pánico. No obstante, mientras la ansiedad y la depresión correlacionan positivamente de forma bivariada con la alteración de rutinas, su influjo sobre aquellas no es significativo en el modelo multivariado. Solo el estrés ejerce un efecto directo sobre las compras por pánico como indirecto, a través del interés en la pandemia y, sobre todo, de la alteración de rutinas. Esto es consistente con los antecedentes teóricos disponibles. El estrés implicaría la activación de la respuesta de "lucha o escape" (fight-or-flight response) que prepara el cuerpo y anticipa la acción ante la situación de amenaza suscitada por la pandemia (Everly \& Lating, 2013).

Dejando de lado la obviedad que la adquisición de alimentos por los medios que sean garantiza la sobrevivencia, las compras por pánico parecen restituir algún sentido de eficacia personal y relativa al resguardo de los seres queridos, 
lo cual vendría a alimentar la sensación de control sobre la propia circunstancia y producir afectos positivos (e.g., satisfacción, tranquilidad) a pesar de la emocionalidad negativa (e.g., síntomas de ansiedad y de depresión) que predomina en el escenario adverso de la pandemia (Folkman \& Moskowitz, 2000; Sim et al., 2020; Yap \& Chen, 2020).

No obstante, la coherencia de los resultados también sería contextual. El protagonismo de la respuesta de estrés en relación con las compras por pánico es consistente con el período en que los datos del estudio fueron recabados. Entonces las medidas de estado de excepción y de cuarentena domiciliar casi alcanzaban el mes de implementación, el avance de la pandemia era exiguo y se preguntó por comportamientos de compra pasados. Asimismo, la muestra en este estudio cuenta con cierto poder adquisitivo (la mayoría trabaja y su ingreso en el hogar les permite llegar a fin de mes sin problemas) y, por tanto, con ciertos recursos sociales y materiales para enfrentar la situación, lo que incluye poder llevar a cabo el tipo de compras que ocupan esta discusión. Es decir, el grado de avance de la pandemia y las medidas para contrarrestarla parecen propiciar una respuesta predominantemente de estrés, resolutiva, enfocada en solucionar problemas, lo que no impide la presencia de síntomas ansiógenos o depresivos, en tanto que afectos negativos caracterizados por la comorbilidad (OPS \& OMS, 2017).

Cabe hipotetizar que, con el paso del tiempo, el avance de la casuística de contagio y los decesos, la presión sobre las finanzas, la tensión de la convivencia forzada y los efectos del encierro prolongado, la manifestación y los niveles de los síntomas emocionales serían distintos, quizás más severos, o el estrés habría dado paso a expresiones más marcadas de ansiedad o depresión (Everly \& Lating, 2013).

\section{CONCLUSIÓN}

Paradójicamente, aunque las compras por pánico constituyen un fenómeno reconocido, su estudio sistemático bastante desconocido, en especial en contextos latinoamericanos, a pesar de constituir una región signada por crisis y catástrofes. Los resultados obtenidos corroboran que durante el avance de la pandemia por COVID-19 en El Salvador tuvieron lugar compras por pánico, así como derivados psicológicos propiciados por la situación de incertidumbre reinante. Al menos un $40 \%$ de la muestra participante habría incurrido en comportamientos propios de compras por pánico. A nivel bivariado, estas correlacionan con síntomas emocionales como la depresión, la ansiedad y el estrés, así como con el interés en el tema de la pandemia y 
la alteración de rutinas. Sin embargo, un análisis de trayectoria mostró que, aunque la depresión y la ansiedad ejercen efectos indirectos sobre las compras por pánico mediados por el interés en el tema, solo el estrés ejerce efectos indirectos y directos sobre dichas compras. Los resultados son congruentes con la literatura especializada en cuanto a la naturaleza diferencial de la depresión, la ansiedad y el estrés en su potencial para catalizar comportamientos e ideaciones activas o pasivas según la circunstancia que configura el avance de la pandemia.

Las limitaciones del estudio remiten a la naturaleza conveniente de la muestra, las mediciones y la circunstancia en que se recabaron los datos. Como fue expuesto, se trata de una muestra no probabilística fruto de un proceso de bola de nieve en línea y cuya cobertura estuvo sujeta a la dinámica de diseminación del cuestionario. Las mediciones realizadas, con excepción de la escala DASS-21, responden a escalas breves construidas ad-hoc para el estudio y al calor de la situación de premura de la pandemia. No obstante, intentar recrear la "tormenta psicológica perfecta" que sugería Novemsky, es decir, incluir constructos psicológicos a granel con el afán de explicar más varianza de las compras por pánico es una tentación tecnocrática que hay que resistir ante el riesgo de la obviedad teórica (i.e., incluir tendencias consumistas, baja tolerancia a la frustración, impulsividad, personalidad ansiosa, etc.).

En cuanto a la circunstancia en que tiene lugar la recogida de información, esta deviene en una limitante inescapable debido a que se trata de un período en que las medidas súbitas de confinamiento para contrarrestar el avance de la pandemia obligan a realizar reacomodos personales, profesionales y logísticos sobre la marcha. Así, para los participantes el llenado del cuestionario no se realizó en condiciones normales a la vez que su contenido les confrontó con temas sensibles. Para los investigadores, la circunstancia obligó a considerar la extensión del cuestionario, a sacrificar preguntas, a la imposibilidad de insistir en la solicitud de distribución o llenado del instrumento, o a tener que condicionar los tiempos de trabajo al ritmo que marcó la normalidad inestable y cambiante que imponían las medidas de confinamiento por la pandemia.

Como recomendación para avanzar la investigación de los temas aquí tratados, se sugiere la realización de mediciones repetidas conforme la pandemia y las medidas de confinamiento avancen (e.g., niveles de síntomas emocionales en distintos momentos de la pandemia). En un mundo ideal, un diseño de panel, en el que las mismas personas dieran cuenta de su situación psicosocial y material, además con el empleo de recursos cualitativos (e.g., diarios, autoreportes) sería iluminador para conocer el impacto acumulado de la pandemia 
o formas emergentes de afrontarla. Asimismo, cabe depurar la escala de compras por pánico, la que fácilmente podría extender sus ítems con el fin de mejorar sus niveles de consistencia interna. Una tercera consideración, es que la perspectiva psicológica suele centrarse en el consumidor y poco en quienes proveen los productos o presencian tales compras. Así, una vía complementaria en el estudio de las compras por pánico sería conocer la psicología del proveedor de productos, de quienes tienen la tarea diaria de acomodar y remplazar mercancía, o de todos aquellos que tienen que atestiguar y gestionar el pánico ajeno al interior de los establecimientos al mismo tiempo que gestionar el propio (e.g., cajeras, vigilantes, otros clientes, etc.).

Por último, cabe decir que el acercamiento a las compras por pánico pone de manifiesto la captura de la atención de la perspectiva psicológica sobre un comportamiento "exuberante" durante la pandemia, comparable en su manifestación a los tumultos consumistas de un black friday. Las compras por pánico solo cobran pleno sentido en el marco de una economía neoliberal en la que los individuos cifran su valía personal, su estatus y su condición de ciudadanos en la adquisición excesiva e incesante de cosas. Las compras por pánico responden a determinantes psicológicos particulares, pero también podrían reflejar comportamientos usuales pero exacerbados por la situación de pandemia, así como vicios antidemocráticos en la sociedad. El desabastecimiento de artículos de primera necesidad replica el habitual agotamiento de productos (e.g., teléfonos móviles) el día de su lanzamiento y la necesidad egocéntrica de ser de los primeros en adquirirlos. Las compras innecesarias pueden desabastecer o generar especulación de precios, así como traducirse en endeudamiento o sobregiro de tarjetas de crédito (con el consiguiente agravamiento de problemas psicológicos). A su vez, las medidas de restricción de venta y consumo han propiciado reacciones similares a la abstinencia de las adicciones; $y$ en varios países se instrumentaliza el comportamiento consumista atizado por el alarmismo de las mismas autoridades para justificar como medidas sanitarias verdaderas restricciones de libertades democráticas.

Una mirada crítica sobre las compras por pánico conduce a preguntarse por cómo se manifiesta el pánico cuando se carece de poder adquisitivo, cómo se manifiesta el pánico del pobre, de esa nueva clase social que constituye el "precariado". La compra por pánico se puede entender como una forma de afrontar el estrés en tanto que respuesta adaptativa a la exigencia del medio. No obstante, aunque resulte ser un comportamiento comprensible en momentos de crisis, la psicología debe ser lo suficientemente lúcida para no ignorar el trasfondo alienante y excluyente en que esta tiene lugar. Se 
vuelve esencial enriquecer el estudio y la problematización de la compra por pánico y de los factores y las emociones que la instigan, sin perder de vista el pánico por la compra. Es decir, la inquietud por la imposibilidad de conseguir lo básico mientras otros sí pueden hacerlo. En estas condiciones, quizás los síntomas depresivos sí propician comportamientos activos pues no queda alternativa. Lo que resulta claro es que aún queda mucho por descifrar del papel de las emociones y del afrontamiento en condiciones de crisis sin la mediación normalizada de prácticas consumistas.

Conflicto de interés: Los autores declaran no tener conflicto de interés alguno en la realización de esta investigación.

Agradecimientos: A quienes ayudaron a empujar la bola de nieve que difundió en línea el cuestionario del estudio, y a los participantes que tuvieron el ánimo para colaborar en medio del encierro inédito y extraño provocado por la pandemia.

Financiamiento: Ninguno

\section{REFERENCIAS}

Antúnez, Z., \& Vinet, E. (2012). Escalas de depresión, ansiedad y Estrés (DASS - 21): Validación de la versión abreviada en estudiantes universitarios chilenos. Terapia Psicológica, 30(3), 49-55. https://scielo.conicyt.cl/pdf/terpsicol/v30n3/art05.pdf

Apóstolo, J. L. A., Figueiredo, M. H., Mendes, A. C., \& Rodrigues, M. A. (2011). Depresión, ansiedad y estrés en usuarios de cuidados primarios de salud. Revista Latino-Americana de Enfermagem, 19(2), 348-353. https://www.scielo.br/pdf/ rlae/v19n2/es_17.pdf

Brafman, O \& Brafman, R. (2008). Sway. The irresstible Pull of Irrational Behavior. New York: Brodway Books.

Clark-Carter, D. (2002). Investigación cuantitativa en Psicología. México: Oxford University Press.

Córdova Macías, R., Rodríguez, M., \& Zechmeister, E. J. (2017). Cultura política de la democracia en El Salvador y en las Américas, 2016/17: Un estudio comparado sobre democracia y gobernabilidad. https://www.vanderbilt.edu/lapop/el-salvador.php

Dar, K.A., Iqbal, N., \& Mushtaq, A. (2017). Intolerance of uncertainty, depression, and anxiety: examining the indirect and moderating effects of worry. Asian Journal of Psychiatry, 29, 129-133. https://doi.org/10.1016/j.ajp.2017.04.017

Everly G. S. Jr. \& Lating, J. M. A (2013). Clinical Guide to the Treatment of the Human Stress Response (3a Ed.) New York: Springer. https://link.springer.com/content/ pdf/10.1007\%2F978-1-4614-5538-7.pdf

Folkman, S. \& Moskowitz, J. T. (2000). Positive affect and the other side of coping. American Psychologist, 55(6), 647-654. https://psych415.class.uic.edu/Readings/ Folkman,\%20Positive\%20affect\%20\&\%20coping,\%20AmPsy,\%202000.pdf

Gallagher, C. E., Watt, M. C., Weaver, A. D., \& Murphy K. A. (2017). "I fear, therefore, I shop!" exploring anxiety sensitivity in relation to compulsive buying. Personality and Individual Differences, 104, 37-42. https://doi.org/10.1016/j. paid.2016.07.023 
García-Rivera, B., Maldonado-Radillo, S. E., \& Ramírez Barón, M. (2014). Estados afectivos emocionales (depresión, ansiedad y estrés) en personal de enfermería del sector salud pública de México. Summa Psicológica UST, 11(1), 65-73. http:// pepsic.bvsalud.org/pdf/summa/v11n1/a06.pdf

Gobierno de El Salvador (2020). Plataforma informativa sobre la COVID-19 del Gobierno de El Salvador. https://covid19.gob.sv/06/04/20

Grisham, J. R., Roberts, L., Cerea, S., Isemann, S., Svehla, J., \& Norberg, M. M. (2018). The role of distress tolerance, anxiety sensitivity, and intolerance of uncertainty in predicting hoarding symptoms in a clinical sample. Psychiatry research, 267, 94-101. https://doi.org/10.1016/j.psychres.2018.05.084

Gutiérrez Quintanilla, J. R., \& Portillo García, C. B. (2013). La ansiedad y la depresión como indicadores de problemas de salud mental en los salvadoreños. Revista Electrónica de Psicología Iztacala, 16(2), 533-557. http://revistas.unam.mx/ index.php/repi/article/view/39965/36368

Hooper, D., Coughlan, J., \& Mullen, M. (2008). Structural equation modelling: Guidelines for determining model fit. Electronic Journal of Business Research Methods, 6(1), 53-60. http://www.ejbrm.com/volume6/issue1

Laboratorio de Psicología Clínica de la Fundación Universitaria Konrad Lorenz (Clinik Lab). (s.f.). Depression Anxiety and Stress Scale-21 (DASS-21). https://blogs. konradlorenz.edu.co/files/dass-21.pdf

La Prensa Gráfica (12 marzo, 2020). Incrementan las compras de pánico en El Salvador por emergencia del coronavirus. https://www.laprensagrafica.com/ incrementan_las_compras_de_pxnico_en_el_salvador_por_emergencia_del_ coronavirus-vf20200312mp4.html

Maheswaran, D., \& Agrawal, N. (2004). Motivational and cultural variations in mortality salience effects: Contemplations on terror management theory and consumer behavior. Journal of Consumer Psychology, 14(3), 213-218. https://doi. org/10.1207/s15327663jcp1403_3

Martín Beristain, C. (1999). Reconstruir el tejido social. Un enfoque crítico de la ayuda humanitaria. Barcelona: Icaria Editorial.

Ministerio de Salud de El Salvador [MINSAL] (2017). Boletín de prensa-MINSAL, ISSS y OPS conmemoran el Día Mundial de la Salud. https://www.salud.gob.sv/archivos/ comunicaciones/archivos_comunicados2017/pdf/boletin_prensa06042017.pdf

Molina, K. (2020, marzo 12). Salvadoreños abarrotan los supermercados en busca de alcohol en gel y productos de limpieza. https://www.elsalvador.com/noticias/ negocios/coronavirus-productos-comerciales-supermercados/694890/2020/

Norberg, M. \& Rucker, D. (2020, March 19). Psychology can explain why coronavirus drives us to panic buy. It also provides tips on how to stop. https://theconversation.com/psychology-can-explain-why-coronavirus-drives-us-to-panic-buy-italso-provides-tips-on-how-to-stop-134032

Novemsky, E. (2020, March 19). Why a Pandemic Leads to Panic Buying. Yale Insights. https://insights.som.yale.edu/insights/why-pandemic-leads-to-panic-buying

Organización Mundial de la Salud [OMS] (2020). Q\&A on coronaviruses (COVID-19). https://www.who.int/news-room/q-a-detail/q-a-coronaviruses

Organización Panamericana de la Salud [OPS]/ Organización Mundial de la Salud [OMS] (2017). Depresión y otros trastornos mentales comunes. Estimaciones sanitarias mundiales. Washington, D.C.: Organización Panamericana de la Salud. https://iris.paho.org/handle/10665.2/34006

Román, F., Vinet, E., \& Alarcón Muñoz, A. (2014). Escalas de Depresión, Ansiedad y Estrés (DASS-21): Adaptación y propiedades psicométricas en estudiantes 
secundarios de Temuco. Revista Argentina de Clínica Psicológica, 23(2), 179-190. https://scielo.conicyt.cl/pdf/terpsicol/v30n3/art05.pdf

Ruiz, F. J., García-Martín, M. B., Suárez-Falcón, J. C., \& Odriozola-González, P. (2017). The hierarchical factor structure of the Spanish version of Depression Anxiety and Stress Scale - 21. International Journal of Psychology and Psychological Therapy, 17, 97-105. https://blogs.konradlorenz.edu.co/files/ruiz2017dass21.pdf

Schreiber, J. B., Nora, A., Stage, F. K., Barlow, E. A., \& King, J. (2006). Reporting structural equation modeling and confirmatory factor analysis results: A review. The Journal of Educational Research, 99(6), 323-337. https://doi.org/10.3200/ JOER.99.6.323-338

Sheu, J. B., \& Kuo, H. T. (2020). Dual speculative hoarding: A wholesaler-retailer channel behavioral phenomenon behind potential natural hazard threats. International Journal of Disaster Risk Reduction, 44, 101430. https://doi.org/10.1016/j. ijdrr.2019.101430

Sim, K., Chua, H. C., Vieta, E., \& Fernandez, G. (2020). The anatomy of panic buying related to the current COVID-19 pandemic. Psychiatry Research, 288, 113015. https://www.ncbi.nlm.nih.gov/pmc/articles/PMC7158779/pdf/main.pdf

Taber, K. S. (2018). The use of Cronbach's alpha when developing and reporting research instruments in science education. Research in Science Education, 48, 1273-1296. https://doi.org/10.1007/s11165-016-9602-2

Taylor, S. (2019). The Psychology of Pandemics: Preparing for the Next Global Outbreak of Infectious Disease. Newcastle upon Tyne: Cambridge Scholars Publishing.

Thomson, W. (2016). Comorbidity between Depression and the Results of Mortality. Journal of Depression \& Anxiety, 5(3), 1-4. https://www.longdom. org/open-access/comorbidity-between-depression-and-the-results-of-mortality-2167-1044-1000236.pdf

Urzúa, A., Vera-Villarroel, P., Caqueo-Urízar, A., \& Polanco-Carrasco, R. (2020). La Psicología en la prevención y manejo del COVID-19. Aportes desde la evidencia inicial. Terapia Psicológica, 38(1), 103-118. https://teps.cl/index.php/teps/ article/view/273

Yap, A.J. \& Chen, C. Y. (2020, March 2). The Psychology Behind Coronavirus Panic Buying. Insead Knowledge, 1-2. https://knowledge.insead.edu/node/13451/pdf

Zimbardo, P. y Boyd, J. (2009). La paradoja del tiempo. Barcelona: Paidos.

Esta obra está bajo: Creative commons attribution 4.0 international license. El beneficiario de la licencia tiene el derecho de copiar, distribuir, exhibir y representar la obra y hacer obras derivadas siempre y cuando reconozca y cite la obra de la forma especificada por el autor o el licenciante.

\section{(cc) BY}

